\title{
Comparative analysis of habitus and behavioral responses in birds in desynchronosis conditions
}

\author{
Tatyana Pogrebnyak ${ }^{1 *}$, Elena Khorolskaya ${ }^{1}$, Oksana Vorobyova $^{1}$, and Irina Sagalaeva ${ }^{2}$ \\ ${ }^{1}$ Department of Biology, Belgorod State National Research University, Belgorod, Russia \\ ${ }^{2}$ Department of Foreign Languages and Professional Communication, Belgorod State National Research University, Belgorod, \\ Russia
}

\begin{abstract}
The dynamics of behavioral reactions is studied. Habit, live weight of roosters in artificially created models of desynchronosis: acute, due to three-day inversion of the light regime, and chronic, due to crowding - increased placing density. An assessment of the behavior, habitus, feed intake, feather cover and molting conditions in combination with the glycemia level, which together provide an objective assessment of the effect of desynchronosis on the functional state of birds and their adaptation to acute and chronic stress.
\end{abstract}

\section{Introduction}

The animal's body always responds to the action of any stressor with stereotypical behavior It is a chain of successive reactions that are genetically determined, they are quickly activated by a complex of neuro-endocrine mechanisms $[1,2,3]$. Functionally, these reactions include a complexly organized hypothalamic-pituitaryadrenal and sympatho-adrenal systems $[4,5]$ Together they act as a system of protection and adaptation on the principle of feedback [6]. A rapid increase in the blood the level of glucocorticoids triggers protective reactions to the stress factor [3, 7]. For example, in the blood of birds, the level of adrenaline, glucogon, corticosteroids, and adrenocorticotropic hormone increases sharply. All these physiological active compounds together cause tachycardia, hypertension in the body, and increase the level of glucose and free fatty acids in the blood [7, 8]. In this state, birds eat less feed, their sexual activity decreases, but against the background of a high level of glycemia, they demonstrate all types of protective behavior $[2,9]$.

In total these reactions with short-term exposure of the stress factor allow birds to get out of stress quickly. Longterm exposure of strong stress factors causes negative processes in birds: hypertrophy of the adrenal and pituitary glands, involution of the lymphoid system with atrophy of the thymus, spleen, and fabricium bag; the production of antibodies decreases, the chemical and cellular composition of blood changes, anabolic reactions slow down, and overall resistance of the body decreases $[2,10]$. In this state, the body of birds cannot exist for a long time. Physiologically, the ability of birds to function at the limit of their physiological capabilities allows them to activate functional and plastic reserves, direct them to strengthen the processes of protein synthesis, stabilize the blood system, and increase the overall non-specific stability of the body. Birds quickly get out of stress along the way of forming an adaptive effect. But only if the stressor was short-lived. Overtension of body functions determines the development of dystrophic and pathological changes in birds with a decrease in productivity and causes the death of birds $[2,11]$.

Stress symptoms in birds do not always appear simultaneously and are often not clearly expressed $[9,10]$. In addition, birds are less sensitive to changes in natural conditions and circumstances of life. However, unnatural conditions always cause stress [2]. The body of birds is able to gradually adapt to long-term environmental stressors [6].

The resistance of the birds' body decreases sharply when a number of components of stereotypical adaptive behavior fall out during the day in conditions of cellular isolation and restriction of living space $[10,11]$.

Stress behavior of birds is genetically determined and includes stereotypical movements. Pronounced vocal agitation, alertness with an elongated neck and raised body, sharp and rapid head movements, panic and fear with vocal responses, threats and erratic movements, depression and lack of movement or, conversely, flight correspond to stress situations and a high level of tension in birds $[9,12]$.

In the course of evolution, the morphofunctional organization of the birds ' body and their adaptive abilities have reached a very high specialization. Despite the research of various aspects of stress in birds, the process of forming adaptive responses involving various brain structures in the implementation of central and peripheral mechanisms of adaptation and disadaptation in stress situations still requires its solution.

\footnotetext{
*Corresponding author: pogrebnyak@bsu.edu.ru
} 
The purpose of the work is to study the features of habitus, daily food and stereotypical behavior in roosters of the white Russian breed in the conditions of experimentally created desynchronosis-acute (photodesynchronosis) and chronic (crowding).

\section{Experimental}

The habit and behavior of 30 mature white Russian roosters aged 1-1. 5 years old were studied in two experimental models of stress when birds were placed in conditions of 30-days desynchronosis. Taking into account that light has a key influence on the rhythm of birds ' life processes, acute stress was simulated by a three-day 12-hour photo-inversion. The shift in the daily photo mode is the strongest non-specific stress factor for birds $[10,13]$. It violates the evolutionary stereotype of endogenous rhythms of body functions due to changes in the natural system of reinforcement of conditioned reflex activity formed during evolution [8, 14].

Light rays are transmitted through the retinodiencephalic connections of the brain to the Hpta nuclei, through the ependyma of the third ventricle of the brainstem to the subcomissural organ and epiphysis, which in birds is the central performer of rhythmic processes during the day. The high sensitivity of chickens to shifts in the photo mode is due to the fact that the centers of the autonomous nervous system, regulation of endocrine glands, and RF tone of the brain stem are localized in the hypothalamus $[15,16]$. The functional activity of these centers is directly controlled by cortical structures, which trigger a stress-tension reaction in birds by autonomous and humoral mechanisms [8]. Daily mode of illumination is the most powerful regulator of their motor activity, eating behavior and feed consumption for birds $[1,12]$.

When preparing animals for the experiment, much attention was paid to handling and pre-training them to the experimental environment and the experimenter. At all stages of the work, the experiments were conducted at the same time of day: the roosters were weighed before feeding and taking blood for analysis from 8.00 to 9.00 o'clock. Blood was obtained by drip from the comb. The level of peripheral blood glycemia was determined by a color reaction with an orthotoluidine reagent $[7,13]$.

The roosters were kept in compliance with the basic zoohygienic norms: in a room with ventilation, in single single-tier wire cages with a mesh floor area of $1350 \mathrm{~cm} 2$, one at a time. The birds had free access to food and water, and received a balanced diet. At the end of each day, the amount of food consumed by the animals was evaluated.

The chronobiological methodological approach was used to form realistic models of neurogenic stress due to a directed change in the daily endogenous and acquired rhythms of behavioral and physiological processes of the body $[13,16]$. The model of chronic stress was created due to the increased density of placing five roosters in a cage for single keeping of a bird (270 $\mathrm{cm}^{2} /$ head) [13]. The severity of the relationship between birds has a linear relationship: the greater is the number of individuals, the higher is the number of antagonistic encounters. In conditions of limited living space, aggressive behavior in birds increases sharply, most often it is accompanied by a beak blow to the side of the trunk of another bird [11].

The habitus of each bird was assessed by the density of the feather cover, the state of the first-order flight feathers on the wings, the degree of expression of stereotypical and emotional-vocal behavior in roosters, as objective indicators of the severity of the stress state $[6$, $11,13]$. In parallel, the live weight of the bird and the amount of food consumed were evaluated. The level of glycemia was determined as an objective correlate of stress $[5,7]$. All these indicators were evaluated before the inversion of the photo mode, after switching the birds to the natural photo mode and crowding on the $1 \mathrm{st}, 3 \mathrm{rd}, 7 \mathrm{th}$, 15 th, 23rd and 30th day [13]. The data obtained in this work were processed using a package of computer programs "Statistica-6".

Research results. During the artificial inversion of the daily photo mode, the daily rhythm of behavior in roosters changed dramatically. Negative emotions and voice responses increased, while at the same time erratic motor activity, anxiety and tension, expressed search and protective-defensive reflexes, especially during the artificial night increased. The subsequent transfer of birds to the natural light mode - from the 1st to the 15th day of the experiment, significantly caused a reduction in the duration of the manifestation of stereotypical behavior associated with feeding and motor activity (head turning, biting, walking, etc.), increased vocal activity, which was accompanied by high aggression and anxiety, fearfulness and fear, the search for threats.

On the 15th-30th days of the experiment, the nature and duration of the stereotypical daily behavior of experienced animals was gradually stabilized. However, the pronounced tremor of the extremities observed from day 3 to day 23, as well as the sudden manifestation of increased alertness, "fading", fast approximate search and aggressive vocal responses indicated high nervous tension, which is usually associated with negative emotions [1].

During the inversion period and the first three days of the experiment, the feed consumption in the experimental group of roosters decreased $(p>0.1)$ (Fig. 1). In the subsequent period - from the 7 th day and by the end of the experiment, feed consumption was gradually stabilized to the initial level. From the 1 st to the 30 th day of adaptive period, the live weight of experimental roosters decreased and at the 30th day it was $6.3 \%(\mathrm{p}>0,1)$ below the baseline (Fig. 2). Its individual fluctuations have been mixed: by the end of the experiment it matches the initial level in three birds, and was below average for $120-350 \mathrm{~g}$ in seven birds.

Starting from the second and especially in the third week of the experiment, all birds had increased loss and breaking of coverts, flight and tail feathers. By the end of the experiment, all experimental roosters were in a state of rapid molting - their wings were missing from two to five or more flight feathers.

Rapid molting indicated the manifestation of a pronounced stress syndrome in birds, which is usually associated with a high level of catabolic hormones in the 
blood, in particular thyroxine, which determines the intensity of metabolic processes [1].

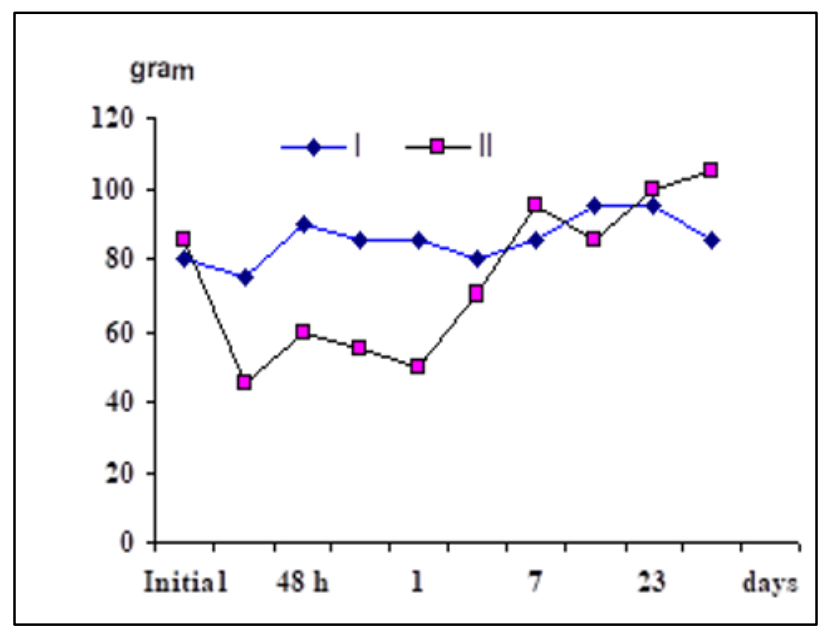

Fig. 1. Dynamics of feed consumption by intact (I) and experimental (II) birds under acute stress.

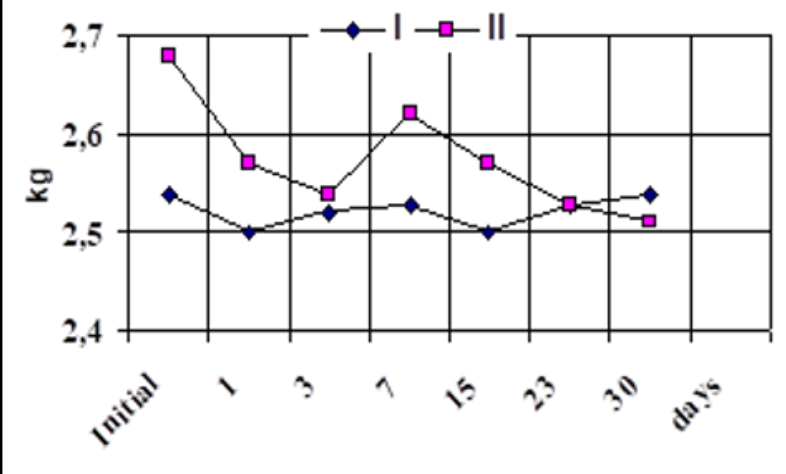

Fig. 2. Dynamics of live weight in intact (I) and experimental (II) birds under acute stress.

A decrease in live weight, a sharp deterioration in feather cover, and a shift in the duration and severity of the daily components of the stereotypical behavior of stressed birds indicated the realism of the created model of acute neurogenic stress, which determined their state of photodesynchronosis.

The nature of stereotypical behavior, the state of feather cover, and changes in live weight in intact birds (control), which were constantly in natural light mode, did not go beyond the background.

In intact roosters during 30 days of observation, the level of glycemia was observed within the physiological norm and ranged from 8.66 to $8.80 \mathrm{mmol} / 1$.

From the beginning of the chronophysiological load after 24 hours, the stressed birds were found to have hyperglycemia, which was $10.75 \pm 0.30 \mathrm{mmol} / \mathrm{l}$. The initial level was exceeded by $26.0 \%(p<0.01)$, after the transfer of stressed birds to the natural photo mode, the level of glycemia decreased sharply and was lower than the background level by $20.5 \%$ for 3 days $(p<0.01)$. On the 15th day, there was a second increase in the level of glycemia, which on the $23 \mathrm{~d}$ day exceeded the background by $30.8 \%$ ( $<<0.01)$, amounting to $11.16 \pm 0.22 \mathrm{mmol} / 1$. A week later, on the 30th day of the experiment, the level of glycemia in experimental birds was shown to be within the initial level. The detected shifts in the level of glycemia in roosters in a state of photodesynchronosis correspond to changes in energy homeostasis in birds under acute neurogenic stress.

The mode of crowding. The increased density of landing experimental birds caused them high nervous and emotional tension from the first minutes which was indicated by aggressive defensive and tentative search reactions, which were accompanied by pecking in the crest and side, threatening vocal responses. The birds stretching their necks often paused, and then performed rapid head movements.

During the 1st day of stress, the birds ' daily behavior changed dramatically with a violation of the duration and nature of their stereotypical behavior related to eating and feather cover care. This pattern of behavior in experimental birds was shown during the first week of crowding, especially during the first three days when hierarchical relationships were established in each cage.

On the 1st day of crowding the most pronounced manifestation of negative emotions was recorded in experimental birds. A sharp restriction of motor activity did not allow them to avoid the negative impact of the created conflict stress situation and to implement the dynamic stereotype, that is, the rhythmicity of the previously formed sequence of daily life processes.

In each cage, the more aggressive roosters became dominant, standing most of the time and often displaying threatening vocal responses with a sharp movement of the beak towards other birds. The roosters with low social status were mostly in the sitting position, not being able to change the position of the body actively. These birds were the most restless and timid, with a constant display of limb tremors and a decrease in body weight in the first half of the crowding period.

Since the 15 th day the stressed birds gradually began to reduce the tense and aggressive nature of behavioral reactions, while increasing the severity of the daily motor mode, the duration of approximate search behavior and stereotypical reactions associated with eating and cleaning feathers. Since the third week of crowding, the feather cover of experimental roosters has significantly deteriorated, as they began the processes of intensive loss and breaking of coverts and flight feathers. Intensive molting was observed in all birds and on the 30th day. Its manifestation as a correlate of a pronounced stress state [2] directly indicated a more pronounced stress in all experimental birds.

In the first week of crowding, especially in the first three days, the feed consumption of experimental roosters decreased sharply ( $p>0.1)$, but in the subsequent period it gradually increased and by the end of the third week slightly exceeded its initial level (Fig. 3). The live weight of experimental roosters decreased directionally during the first three weeks of crowding and on the 23rd day was minimal-14.9\% ( $\mathrm{p}>0.1)$ below the background.

On the 30th day, the live weight of roosters was somewhat stabilized, but compared to the original was 6.8 $\%$ (p>0.1) lower (Fig. 4). 
Since the 15th day of crowding, there has been a tendency to approach the initial level of stereotypical behavior and live weight of experimental birds. The obtained data can be considered as indicators of the body's output to a certain level of adaptation to a state of chronic stress, since by this time the birds had established hierarchical relationships in each cage and, accordingly, reduced nervous and emotional tension.

During the entire 30-days study period, the indicators of the overall functional state of intact roosters (stereotypical behavior, live weight and feed consumption, feather cover condition) did not go beyond the background values.

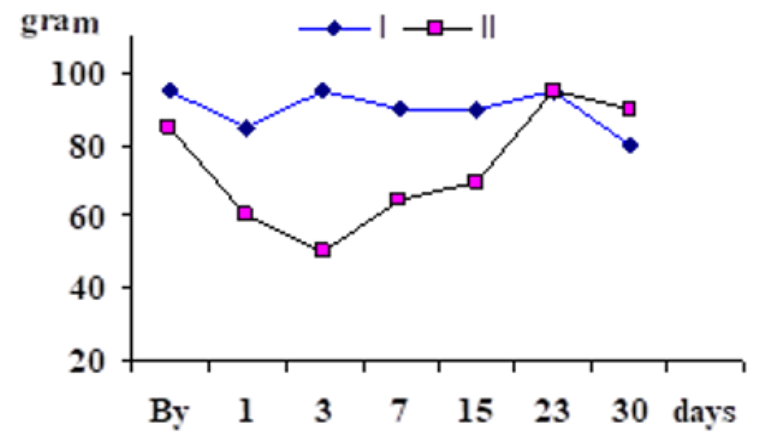

Fig. 3. Feed consumption of intact (I) and stressed (II) birds under conditions of chronic stress.

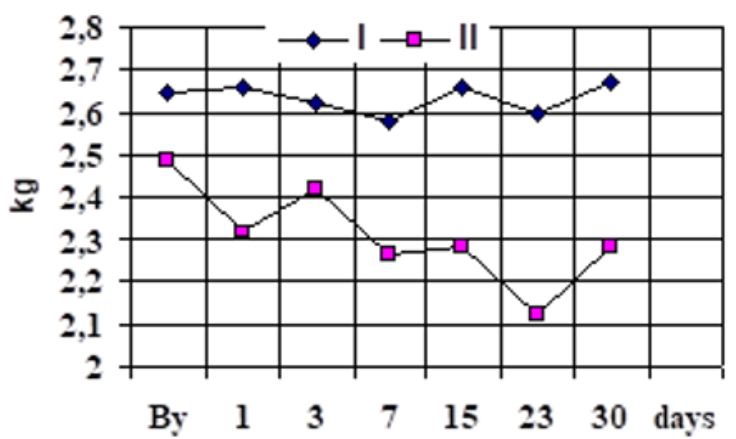

Fig. 4. Dynamics of live weight in intact (I) and experimental (II) birds under conditions of chronic stress.

\section{Results and discussion}

The loss of a number of components of stereotypical behavior in birds that are the result of an evolutionary process during intensive poultry farming does not allow them to fully adapt to relatively constant industrial conditions. The cage placing of birds with a sharp restriction of motor activity associated with the search for food, deform the evolutionarily formed daily stereotypical behavior of domestic birds and associated cyclic rhythms of physiological functions of the body, violating the mechanisms of homeostasis regulation.

To assess the functional state of birds under acute and chronic stress, along with the dynamics of the level of glycemia it is necessary to study the dynamics of changes in the habit of birds including the state of feather cover and the rate of development of molt and pronounced behavioral responses under stress. A longer observation period is needed to study crowding stress.

\section{References}

1. A.A. Viru, Usp. fiziol. Sciences. 11, 27 (1980)

2. Yu.P. Kvitkin, N.G. Federchenko, I.L. Krivtsov, Stress of poultry. Overview information. (1977)

3. S. Levine, Animal Behav. 217 (1976)

4. T. Cox, Stress. (1981)

5. F.I. Furduy, V.P. Fedoryak, S.Kh. Haidarliu. Chisinau: Shtiintsa. (1987)

6. W.J. Kuenzel, Proceedings. 75 (1982)

7. L.E. Panin, Biochemical mechanisms of stress. (1983)

8. F.P. Vedyaev, T.M. Vorobyo, Models and mechanisms of emotional stress. (1983)

9. B. Novitsky, The behavior of farm animals. (1981)

10. M. Mathew, Poultry Gicide. 18, 59 (1981)

11. A.M. Evstratova, Ways to increase the viability of poultry in industrial conditions. (1979)

12. V.V. Matyushnikov, Natural resistance of poultry. (1985)

13. T.A. Pogrebnyak, T.M. Vorobyova, E.A. Lipunova, Methods of studying the central nervous mechanisms of adaptation of birds to stress. (1990)

14. P.K. Anokhin, Nodal questions of the theory of a functional system. (1980)

15. F.P. Vedyaev, T.M. Vorobeva, Models and mechanisms of emotional stress. (1983)

16. I.A. Bolotnikov, V.S. Mikhneva, E.K. Oleinik, Stress and immunity in birds. (1983) 